\title{
Visibilidad científica y académica en la web 2.0

\author{
Análisis de grupos de investigación de la \\ Universidad de La Sabana
}

(1) Camilo Alejandro Corchuelo Rodríguez

Centro de Tecnologías para la Academia (CTA), Universidad de La Sabana, Colombia/

camilocoro@unisabana.edu.co / Orcid ID: https://orcid.org/oooo-ooo2-5489-66o2

\begin{abstract}
Resumen
El modelo tradicional de comunicación científica centra la divulgación de resultados en medios tradicionales como revistas, congresos, reportes, conferencias, etc. Sin embargo, con la llegada de la denominada web 2.0 surgen nuevas posibilidades para mejorar la visibilidad de contenidos académicos en espacios de interacción de Internet y nuevas formas de medir su impacto como las denominadas Altmetrics (Priem, 2010). En consecuencia, el objetivo de este estudio fue diagnosticar el estado de la visibilidad de los investigadores de los grupos de investigación de la Universidad de La Sabana en la web 2.o. Para ello se realizó un análisis cuantitativo del tipo descriptivo sobre once plataformas (académicas y sociales) que permiten potenciar la circulación de contenidos científicos y/o académicos en Internet. De esta manera fue posible identificar la baja participación de los investigadores en las plataformas que potencian la visibilidad científica en la web 2.0.
\end{abstract}

\begin{abstract}
Scientific and academic visibility in the web 2.0: analysis groups of research of the University of La Sabana. The traditional model of scientific communication focuses on the dissemination of results in traditional media such as magazines, congresses, reports, conferences, etc. However, with the arrival of the Web 2.0 web, new possibilities have emerged to improve the visibility of academic contents in Internet interaction spaces and new ways to measure their impact, such as the so-called Altmetrics (Priem, 2010). Consequently, the objective of this study was to diagnose the status of the visibility of the researchers of the research groups of the University of La Sabana in web 2.0. For this, a quantitative analysis of the descriptive type was carried out once the academic and social platforms allow the circulation of scientific and / or academic contents on the Internet. In this way it is possible to identify the low participation of researchers in the platforms that enhance scientific visibility in the web 2.0.
\end{abstract}

\section{Palabras clave}

Visibilidad científica Métricas alternativas Redes sociales Redes académicas

Keywords

Scientific visibility Altmetrics Social networks Academic networks 


\section{Introducción}

“La ciencia que no se ve, no existe” (Redalyc, 2008)

\subsection{Divulgación y visibilidad de la producción científica y académica}

El modelo tradicional de la comunicación científica propuesto por Garvey y Griff citado por (Charum et al., 2003) centra la divulgación de resultados de investigación en medios tradicionales como revistas, congresos, reportes, conferencias, entre otros. Bajo este modelo países como Colombia registran una producción científica baja, aportando un $0,2 \%$ a la producción mundial, manteniendo niveles inferiores de indexación, difusión y divulgación que relegan al país en plataformas de análisis de impacto de producción científica como Scimago (Colciencias, 2016).

En el marco académico las universidades son en esencia las instituciones que mayor conocimiento generan en la sociedad (Codina, 2016). En este sentido, Redalyc (2008) considera fundamental en la labor científica la visibilidad de los resultados y/o avances de la investigación, de esta forma se crean nuevos conocimientos que permiten la construcción colectiva de nuevos saberes. Por tal motivo, la visibilidad del conocimiento se concibe como la capacidad de hacer disponible la producción intelectual a la comunidad científica en diferentes medios y/o soportes, permitiendo aumentar la calidad de las publicaciones (Turpo y Medina, 2013; González et al., 2011). Además, con la masificación de Internet conseguir una mayor visibilidad no significa necesariamente en un mayor impacto científico (citas) pero sí en un aumento directo en la utilización de los contenidos producidos (Torres-Salinas y Milanés-Guisado, 2014).

Diferentes estudios han analizado la visibilidad de la producción científica de las instituciones de educación superior. Iribarren (2006) estudió los investigadores de la Universidad Carlos III entre 1997 y 2003, y centró el análisis en los contenidos indexados en la plataforma ISI Web of Knowledge. Como resultado se identificó la necesidad de mejorar los contenidos indexados en esta base de datos, mediante estrategias como la diversificación de tipologías documentales, el registro riguroso de las referencias de los documentos y el correcto registro de los autores. Este último, es un problema que afecta la identificación de la producción científica y la medición del Índice H de los autores (Corchuelo-Rodríguez, 2013). Un estudio similar realizado por González et al., (2011) en la Universidad de Central Abreu analizó la visibilidad de la producción científica de esta institución en el período de 2000 a 2008 en la base de datos ISI Web of Knowledge, identificando un factor de impacto bajo de las publicaciones, poco nivel de citación de

1. Scielo (Scientific Electronic Library Online) es una biblioteca electrónica que abarca una colección seleccionada de revistas científicas brasileñas. los autores y una destacada visibilidad de los contenidos generados en conferencias y congresos. Finalmente, Alperin (2016) analizó la visibilidad de la colección de la base de datos Scielo ${ }^{1}$ Brasil, identificando en el gestor bibliográfico Mendeley un bajo registro de artículos (<50\%) y menos de una mención por artículo en las redes sociales Facebook y Twitter.

\subsection{Visibilidad de la producción científica y académica en la web 2.0}

Con el surgimiento de la denominada web 2.0 los usuarios de Internet adoptan un papel activo en la generación y difusión de información, cambiando el paradigma de los medios tradicionales de comunicación (O'Reilly, 2005). En consecuencia, surgen nuevas posibilidades para visibilizar contenidos académicos en espacios de interacción como redes sociales, redes académicas, plataformas científicas, entre otros. En este contexto, la comunidad científica y académica del mundo 
ha encontrado una oportunidad para divulgar y visibilizar contenidos mediante bases de datos, repositorios, blogs, wikis, gestores bibliográficos, entre otros, que facilitan la trasferencia de conocimiento de la academia a la sociedad. Con el fin de analizar el impacto de estas nuevas dinámicas surgen las denominadas métricas alternativas o Altmetrics (Priem, 2010), cuyo propósito es analizar la visibilidad web de la ciencia en las aplicaciones y plataformas sociales de la web 2.0. Para Alonso-Arévalo y Vázquez estas mediciones permiten recopilar información de diversas fuentes de información como medios de comunicación, blogs, redes y medios sociales, lo que presenta una oportunidad para "la visibilidad, identidad y reputación digital de los autores y sus obras. Los sistemas altmétricos rastrean y compilan información de una amplia variedad de fuentes que dan una idea más cercana al impacto real de la investigación" (Alonso-Arévalo y Vázquez, 2016: 18).

\section{3 ¿Cómo se mide la visibilidad de la producción científica y académica en la web 2.0?}

De acuerdo con Alperin (2016), Borrego (2014) y Lin y Fenner (2013) se han creado diferentes plataformas para la medición y seguimiento de las Altmetrics. A continuación se presentan las tres herramientas ${ }^{2}$ que de acuerdo con la literatura tienen mayor alcance e impacto en la medición de estas métricas.

» Plumanalytics: Esta plataforma tiene como característica principal la integración de las métricas de consulta y accesos a las plataformas EBSCO y PLOS, Pubmed y Scopus, de esta manera se integran cinco categorías que incluyen mediciones tradicionales y actuales (Social Media Metrics, Usage Metrics, Capture Metrics, Mention Metrics y Citation Metrics).

» Impactstory: De acceso gratuito, permite analizar 6 plataformas de redes sociales y académicas. Dentro de sus características se destaca la integración de dos gestores bibliográficos (Mendely y CrossRef) como fuente para la consulta de métricas de citación.

»Altmetric: Tomó el nombre de las nuevas analíticas emergentes; esto ha generado confusión en parte de la comunidad académica al relacionar el término directamente con esta plataforma. Es la herramienta de mayor impacto y alcance en métricas alternativas; integra fuentes de información académicas como Scopus; además, explora nuevos elementos en la visibilidad web como noticias, blogs y publicaciones en Wikipedia.

\section{4 ¿Cómo mejorar la visibilidad en la web 2.0?}

Luego de triangular los indicadores ${ }^{3}$ de análisis de las plataformas de mayor impacto en la medición de las métricas alternativas a nivel mundial Plumanalytics, Almetric e Impactstory con las redes de mayor impacto y crecimiento en los últimos 5 años en Internet (Cosenza, 2017; IRedes, 2017), emergen las redes y plataformas (5 redes sociales y 6 académicas) con mayor visibilidad para la producción científica y académica en la web 2.0 (Tabla I).

\section{Materiales y métodos}

Enfoque de la investigación cuantitativo y diseño descriptivo. De esta manera, se realizó un proceso riguroso de recolección de datos de acuerdo con las variables establecidas (Briones, 2003; Mora, 2006; Namakforoosh, 2000). A continuación se describen los elementos metodológicos que caracterizan la investigación.
2. https://plumanalytics. com/, https://impactstory.org $y$ https://www.altmetric.com

3. Los indicadores de las tres plataformas se encuentran documentadas en http:// camicorchuelo.wixsite.com/ unisabanainvestiga/altmetrics 
Tabla I. Redes sociales y académicas con mayor impacto para aumentar la visibilidad científica y académica en la Web 2.0

\begin{tabular}{|l|l|l|}
\hline Tipo de red & Nombre & URL \\
\hline Red social & Linkedln & https:/www.linkedin.com \\
\hline Red social & YouTube & https://www.youtube.com \\
\hline Red social & Twitter & https://twitter.com/ \\
\hline Red social & Facebook & https://www.facebook.com \\
\hline Red social & Google + & https://plus.google.com/u/o \\
\hline Red académica & ORCID & https://orcid.org/ \\
\hline Red académica & Google Scholar & https://scholar.google.es/ \\
\hline Red académica & Mendeley & https://www.mendeley.com/ \\
\hline Red académica & Academia.edu & https://www.academia.edu/ \\
\hline Red académica & MyScienceWork & https://www.mysciencework.com/ \\
\hline Red académica & ResearchGate & https://www.researchgate.net/ \\
\hline
\end{tabular}

\subsection{Muestra y población}

La población de la investigación comprende los 49 grupos de investigación de la Universidad de La Sabana. Sin embargo, de acuerdo con Moreno (1999) en este tipo de estudios es necesario establecer una muestra representativa de análisis; en consecuencia, mediante un muestreo aleatorio se seleccionaron 9 grupos de investigación.

\subsection{Variables de análisis}

En estudios cuantitativos las variables se convierten en propiedades cuya variación puede ser medible y observable (Hernández-Sampieri; Fernández Collado y Baptista Lucio, 2014). La tabla II describe las variables usadas en la investigación.

\subsection{Recolección de datos}

La figura 1 muestra el proceso de recolección de datos realizado para el desarrollo de la investigación.

\section{Resultados y discusión}

\subsection{Muestra}

El diagnóstico se aplicó a 101 investigadores activos de 9 grupos de investigación de la Universidad de La Sabana. El grupo de investigación con mayor representación

Búsqueda de

información en las

redes sociales y académicas
Análisis de los datos 


\section{Descripción}

$\begin{array}{ll}\text { Variables } & \text { VI: Investigadores activos de los grupos de investigación de la } \\ \text { Universidad de La Sabana } \\ \text { VD: } \\ \text { » Redes sociales (Linkedln, YouTube, Twitter, Facebook, Google +) } \\ \text { " Redes académicas (ORCID, Google Scholar, Mendeley, Academia. } \\ \text { edu, MyScienceWork, ResearchGate) }\end{array}$

VE: Los investigadores activos de la universidad tiene alta visibilidad en la web 2.0

Control de Se tendrán en cuenta los perfiles de las redes sociales y variables académicas de cada investigador activo y su perfil de CVLAC ${ }^{2}$.

Hipótesis Hi: Los investigadores activos de los grupos de investigación de la Universidad de la Sabana tienen alta visibilidad en las web 2.o. Ho: Los investigadores activos de los grupos de investigación de la Universidad de la Sabana tienen NO alta visibilidad en las web 2.0.

Tabla II. Variables de análisis de la investigación

VI: variable independiente

VD: variable dependiente

VE: variable extraña

CVLAC (Currículum Vitae de Latinoamérica y del Caribe): registro único de autor válido para Colciencias en el marco

del Sistema Nacional de Ciencia Tecnología e Innovación - SNCTI. (Corchuelo-Rodriguez \& Devia-Gonzalez, 2018). Hi: hipótesis de investigación

Ho: hipótesis nulas

en el estudio fue Procesos Agroindustriales Unisabana (18\%) y el de menor Trauma y Rehabilitación (4\%) (Tabla III).

\begin{tabular}{|l|l|c|}
\hline Facultad & Grupo de investigación & $\begin{array}{c}\text { \# de } \\
\text { investigadores }\end{array}$ \\
\hline Centro de Tecnologías para la Academia & Proventus & 14 \\
\hline Facultad de Medicina & Centro de Investigación Biomédica & 10 \\
\hline Escuela Internacional de Ciencias Económicas & Negocios, Economía y Finanzas & 12 \\
\hline Escuela Internacional de Ciencias Económicas & Centro de Investigaciones de la Comunicación & 7 \\
\hline Facultad de Derecho y Ciencias Políticas & Derecho Internacional & 7 \\
\hline Facultad de Educación & Educación y Educadores & 18 \\
\hline Clínica Universidad de La Sabana & Trauma y Rehabilitación & 4 \\
\hline Facultad de Ingeniería & Procesos agroindustriales Unisabana & 19 \\
\hline Facultad de Psicología & Cognición, Aprendizaje y Socialización & 10 \\
\hline
\end{tabular}


4. GrupLAC (Grupos de Investigación de Latinoamérica y del Caribe), plataforma para el registro de la información de los grupos de investigación avalados por Colciencias en el marco del Sistema Nacional de Ciencia Tecnología e Innovación - SNCTI. (Colciencias, 2017)

\subsection{Procedimiento}

El proceso metodológico para la realización del diagnóstico inició con la identificación de los grupos de investigación reconocidos por Colciencias en la plataforma del GrupLAC $^{4}$ (Colciencias, 2017). Posteriormente se realizó la búsqueda de cada investigador activo en cada grupo en las redes sociales y académicas de mayor impacto en visibilidad científica y académica en la Web 2.0. Se realizaron aproximadamente 4.000 búsquedas combinando nombres, apellidos e iniciales. Finalmente, se realizó el registro sistemático de los datos en el instrumento elaborado.

\subsection{Visibilidad en redes sociales}

En promedio el $68 \%$ de los investigadores no tiene en cuenta estas plataformas. La red social donde hay mayor registro de investigadores es LinkedIn (60\%) y la plataforma con menor representación es YouTube (12\%)(Figura 2). Por otra parte, el grupo de investigación con mayor presencia de investigadores en estas plataformas es Proventus (23\%), Escuela Internacional de Ciencias Económicas y Administrativa (20\%) y Facultad de Educación (18\%). Por su parte, las facultades con menor representación de investigadores en estas redes son la Facultad de Medicina (5\%) y Facultad de Psicología (5\%) (Figura 3). Finalmente, los investigadores con registro en todas las redes son Suanny Pinzón Rodriguez (Proventus), Loly Aylú Gaitán Guerrero (Negocios, Economía y Finanzas) y Andrés Chiappe Laverde (Proventus) (Tabla IV).

\subsection{Visibilidad en redes académicas}

En promedio el $73 \%$ de los investigadores no tiene cuenta en estas plataformas. La red académica donde hay mayor representación de investigadores es ResearchGate (41\%) y la menor Academia.edu (22\%) y Mendeley (23\%) (Figura 4). Por otra parte, los grupos de investigación con mayor presencia de investigadores son Proventus (24\%) y Educación y educadores (19\%). Las facultades con menor representación en estas redes son Clínica (2\%) y Facultad de Derecho y Ciencias Políticas (3\%) (Figura 5). Finalmente, los investigadores con registro en todas las redes son Cristina Henning Manzuoli (Proventus), Clelia Pineda Báez (Educación y Educadores), Oscar Rafael Boude Figueredo (Proventus) y Andrés Chiappe Laverde (Proventus) (Tabla V).

\section{Conclusiones}

De acuerdo con el concepto de visibilidad en la web 2.0 de Priem (2010) la representación de los investigadores de la Universidad de La Sabana en las redes sociales y académicas de mayor impacto en visibilidad para la producción científica y académicas es baja. En las redes sociales en promedio tan solo el 32\% de los investigadores tiene presencia; las plataformas con menor uso son YouTube (12\%) y Twitter (26\%). Un hecho particular teniendo en cuenta que los estudios de consumo digital identificaron estas como unas de las más usadas por los colombianos (Ministerio de las Tecnologías de la Información y la Comunicación, 2012) (El consumo, 2014). En las redes académicas solo el $27 \%$ de los investigadores tienen registros. Las redes con menor presencia son Academia.edu (22\%), Mendeley (23\%) y Google Scholar (24\%), en esta última resulta un hecho curioso dado que el repositorio institucional de la Universidad de La Sabana donde se deposita la producción científica y académica de todos los grupos de investigación indexa sus contenidos en esta plataforma. Además, es considerado por varios autores como el buscador académico universal, con mayor facilidad de uso para docentes e investigadores (Carreño, 2017; Orduña, Martín, Ayllón y López, 2016). 


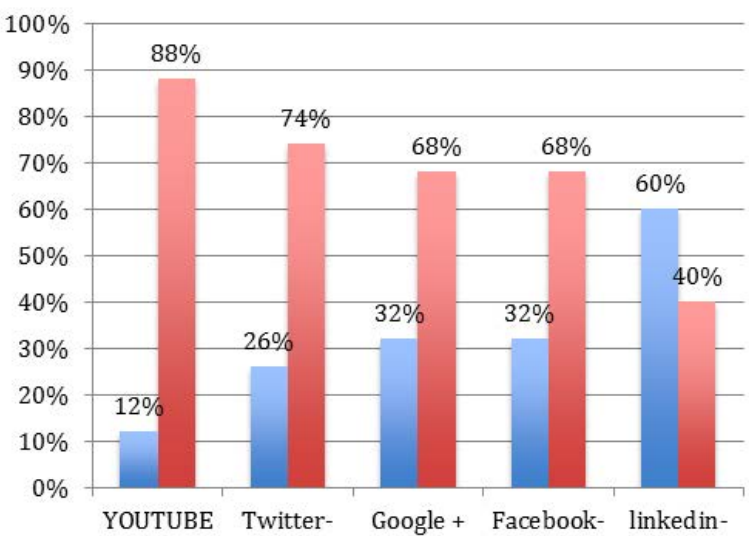

- Investigadores registrados

- Investigadores sin registro

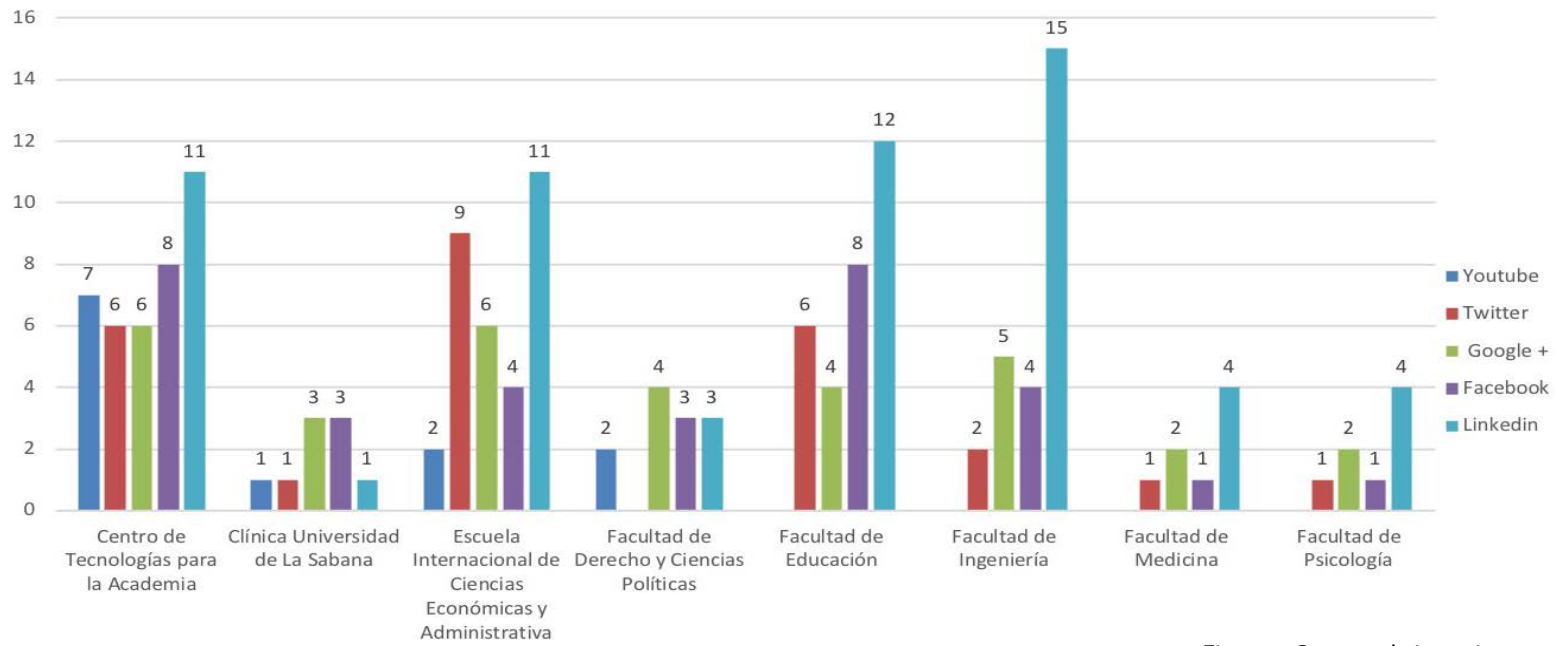

Figura 3. Cuentas de investigadores en las redes sociales de mayor visibilidad para la producción científica y académica en la web 2.o. por facultad.

\begin{tabular}{|l|l|c|}
\hline Grupo de investigación & Investigador & $\begin{array}{c}\text { Número } \\
\text { de perfiles }\end{array}$ \\
\hline Proventus & Suanny Pinzón Rodriguez & $5 / 5$ \\
\hline Negocios, Economía y Finanzas & Loly Loly Aylú Gaitán Guerrero & $5 / 5$ \\
\hline Proventus & Andrés Chiappe Laverde & $5 / 5$ \\
\hline $\begin{array}{l}\text { Centro de Investigaciones de } \\
\text { la Comunicación Corporativa } \\
\text { Organizacional }\end{array}$ & Ángela Sofía Preciado Hoyos & \\
\hline Trauma y Rehabilitación & Juan Guillermo Ortiz Martinez & $4 / 5$ \\
\hline Derecho Internacional & Maria Carmelina Londoño Lázaro & $4 / 5$ \\
\hline Negocios, Economía y Finanzas & Anne Marie Zwerg Villegas & $4 / 5$ \\
\hline Proventus & Olga González Sosa & $4 / 5$ \\
\hline Educación y Educadores & Jefferson Galeano Martinez & $4 / 5$ \\
\hline Educación y Educadores & Tatiana Ghitis Jaramillo & $4 / 5$ \\
\hline
\end{tabular}

Tabla IV. Top 10 de los investigadores con más perfiles en las redes sociales de mayor visibilidad para la producción científica y académica en la web 2.0

Figura 2. Investigadores con cuenvisibilidad para la producción científica y académica en la web 2.0 
Figura 4. Investigadores con cuentas en las redes acadé-

micas de mayor visibilidad para la producción científica y académica en la web 2.0 .

Figura 5. Cuentas de investigadores en las redes académicas de mayor visibilidad para la producción científica y académica en la web 2.o. por facultad

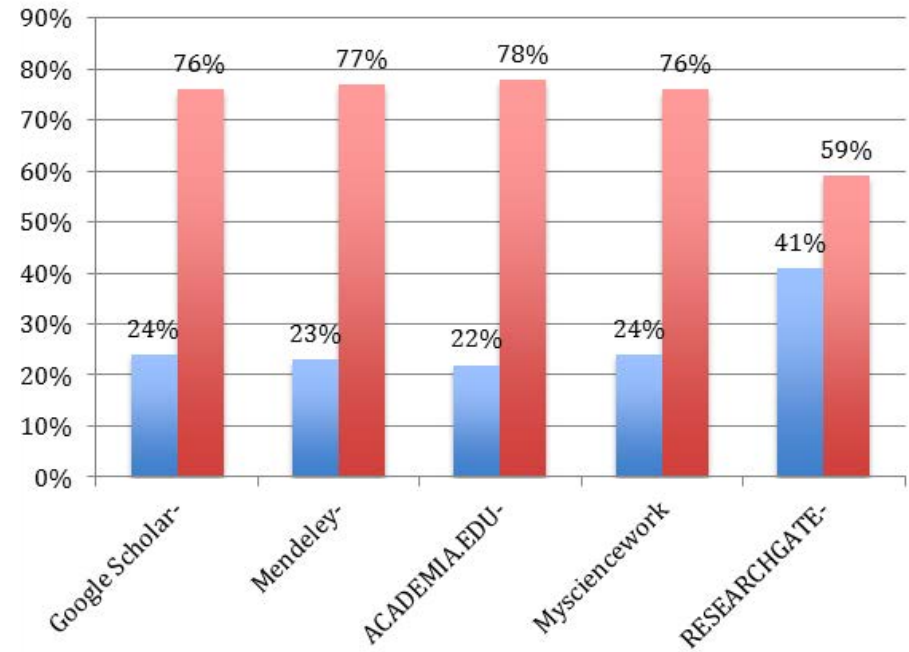

- Investigadores registrados

Investigadores sin registro

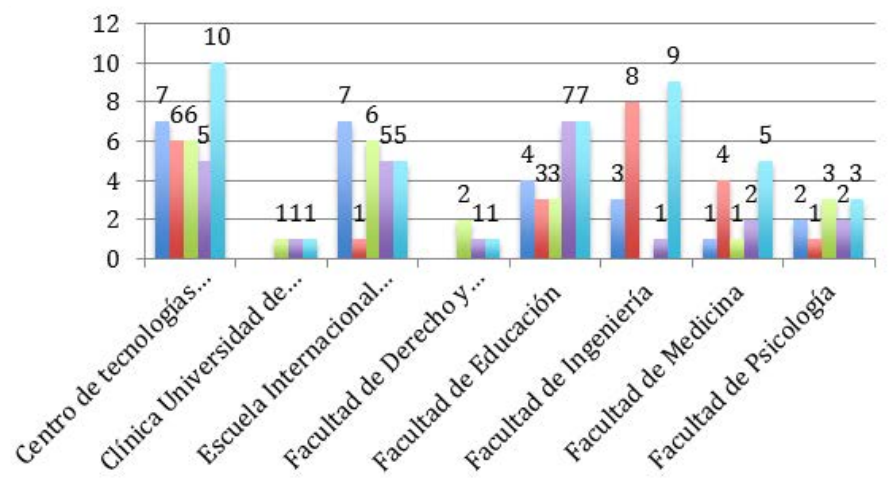

= Google Scholar-

- Mendeley-

ACADEMIA.EDU-

- Mysciencework

- RESEARCHGATE-

\begin{tabular}{|l|l|c|}
\hline Grupo de investigación & Investigador & $\begin{array}{c}\text { Número de } \\
\text { perfiles }\end{array}$ \\
\hline Proventus & Cristina Hennig Manzuoli & $6 / 6$ \\
\hline Educación y Educadores & Clelia Pineda Báez & $6 / 6$ \\
\hline Proventus & Oscar Rafael Boude Figueredo & $6 / 6$ \\
\hline Proventus & Andrés Chiappe Laverde & $6 / 6$ \\
\hline Negocios, Economía y Finanzas & Anne Marie Zwerg Villegas & $6 / 6$ \\
\hline Educación y Educadores & Tatiana Ghitis Jaramillo & $5 / 6$ \\
\hline Proventus & Hugo Alexander Rozo Garcia & $5 / 6$ \\
\hline Negocios, Economía y Finanzas & Catherine Pereira Villa & $5 / 6$ \\
\hline $\begin{array}{l}\text { Centro de Investigación } \\
\text { Biomédica Universidad de La } \\
\text { Sabana (CIBUS) }\end{array}$ & Fernando Lizcano Losada & \\
\hline $\begin{array}{l}\text { Procesos Agroindustriales } \\
\text { Unisabana }\end{array}$ & Annamaria Filomena Ambrosio & $4 / 6$ \\
\hline
\end{tabular}

Tabla V. Top 10 de los investigadores con más perfiles en las redes académicas de mayor visibilidad para la producción científica y académica en la web 2.o. 
De acuerdo con lo anterior, Alperin (2015) indica que en América Latina los porcentajes de divulgación y uso de plataformas web 2.0 por los autores es bajo. Además, los investigadores no tienen la cultura de compartir el conocimiento que producen. En este sentido, la Universidad de La Sabana en el ranking de universidades QS desde el 2014 mantiene su ubicación entre el \#67 (2014) y el \#65 (2016), registrando los puntajes de calificación más bajos en citación por documento y artículos por facultad. Sin embargo, estas cifras no son consecuentes con la producción científica y académica de sus 49 grupos de investigación(«QS Top Universities», 2015). Por tal motivo, el diagnóstico permite evidenciar falta de divulgación y visibilidad de la producción científica por parte de los investigadores en las redes sociales y académicas de mayor impacto. En este sentido, los investigadores deben ver el uso de las redes sociales y académicas indicadas en el estudio como una oportunidad para distribuir y compartir su producción intelectual, aumentando su posicionamiento web (SEO académico), mejorando su identidad digital, incrementando la probabilidad de que sus documentos sean consultados, descargados y/o citados, entre otros (Lin y Fenner, 2013; Borrego, 2014; Codina, 2016; Alonso-Arévalo y Vázquez Vázquez, 2016). 


\section{Q Referencias bibliográficas}

》Alonso-Arévalo, J. y M. Vázquez Vázquez. 2016. Altmetrics y alfabetización científica. En Bibliotecas anales de investigación. Vol. 12, no. 1, 14-29.

"Alperin, J. P. 2015. Geographic variation in social media metrics: an analysis of Latin American journal articles. En Aslib Journal of Information Management. Vol. 67, no. 3, 289-304. <https://doi.org/10.1108/AJIM-12-2014-0176> [Consulta: 30 marzo 2018].

»Alperin, J. P. 2016. \#Aprender3C - ¿Qué impacto miden las métricas alternativas en publicación científica? <https://www.youtube.com/watch?v=Usc47462zbo> [Consulta: 30 marzo 2018].

"Borrego, A. 2014. Altmétricas para la evaluación de la investigación y el análisis de necesidades de información. En El Profesional de la Información. Vol. 23, no. 4, 352-357. <https://doi.org/10.3145/epi.2014.jul.o2> [Consulta: 30 marzo 2018].

»Briones, G. 2003. Metodología de la investigación cuantitativa en las ciencias sociales (Vol. Módulo 3). Bogotá: ARFO. <https://metodoinvestigacion.files.wordpress.com/2008/02/metodologia-de-la-investigacion-guillermo-briones.pdf> [Consulta: 30 marzo 2018].

"Carreño, R. 2017. La revolución Google Scholar: destapando la caja de Pandora académica. En Anales de Documentación. Vol. 20, no. 1. <http://revistas.um.es/ analesdoc/article/view/289361>[Consulta: 30 marzo 2018].

»Charum, J.; C. Murcia; D. Usgame; A. Silva; C. Barbosa y S. Rodríguez. 2003. La búsqueda de la visibilidad a través de la calidad: el reto del editor. Bogotá: ICFES.

»Codina, L. 2016. Difusión de la investigación: Open Access, repositorios y redes sociales. <http://repositori.upf.edu/handle/10230/27515> [Consulta: 30 marzo 2018].

"Colciencias. 2016. Políticas para mejorar la calidad de las publicaciones científicas nacionales. <http://www.colciencias.gov.co/sites/default/files/upload/paginas/ politica-publindex-colciencias.pdf> [Consulta: 30 marzo 2018].

"Colciencias. 2017. GrupLAC [Guvernamental]. <http://scienti.colciencias.gov. co:8083/ciencia-war/busquedaGruposPorlnstitucion.do> [Consulta: 30 marzo 2018].

»El consumo digital en Colombia. 2014. En Revista Diners. Actualidad. 20 de febrero 2014. <http://revistadiners.com.co/actualidad/13179_el-consumo-digital-en-colombia/> [Consulta: 7 febrero 2017].

"Corchuelo-Rodríguez, C. A. 2013. Bibliometría: análisis del índice: los indicadores de autor y su aplicación en la comunidad científica colombiana. Universidad de la Salle, Bogotá, Colombia. Trabajo de grado. <http://eprints.rclis.org/24678/> [Consulta: 30 marzo 2018].

»Cosenza, V. 2017. World map of social networks [Blog]. <http://vincos.it/worldmap-of-social-networks/> [Consulta: 15 julio 2017].

» González, P.; M. Josefa; S. Cabrera; F. Manuel; P. Suárez y L. Manuel. 2011. Visibilidad e impacto de la producción científica de la Universidad Central «Marta Abreu» de Las Villas durante el período 2000-2008. En ACIMED. Vol. 22, no. 1, 6o-78. 
» Hernández-Sampieri, Roberto; Carlos Fernández Collado y María del Pilar Baptista Lucio. 2014. Metodología de la investigación. McGraw-Hill Interamericana.

"IRedes. 2017. Mapa IRedes. IRedes. <http://www.iredes.es/mapa/> [Consulta: 15 julio 2017].

" Iribarren, I. 2006. Producción científica y visibilidad de los investigadores de la Universidad Carlos III de Madrid en las bases de datos del ISI, 1997-2003. Universidad Carlos III. Getafe. Tesis de Doctorado. <http://orff.uc3m.es/bitstream/ handle/10016/1088/TESIS\%20lIM.pdf? sequence $=1>$ [Consulta: 15 julio 2017].

»Lin, J. y M. Fenner. 2013. Altmetrics in evolution: Defining and redefining the ontology of article-level metrics. En Information Standards Quarterly.Vol. 25, no. 2, 20. <https://doi.org/10.3789/isqv25no2.2013.04> [Consulta: 15 julio 2017].

» Ministerio de las Tecnologías de la Información y la Comunicación, Colombia. 2012. 8 de cada 10 colombianos están usando Internet. Ministerio de Tecnologías de la Información y las Comunicaciones. <http://www.mintic.gov.co/portal/604/w3-article-1629.html> [Consulta: 7 febrero 2017].

» Mora, M. E. de la. 2006. Metodología de la investigación: desarrollo de la inteligencia. Thomson.

»Moreno, A. 1999. Recolección de la información Módulo 3. Santafé de Bogotá: ICFES.

»Namakforoosh, M. 200o. Metodología de la investigación. za ed. México: Editorial Limusa ; Grupo Noriega Editores.

» Orduña, E.; A. Martín; J. Ayllón y E. López. 2016. La revolución Google Scholar. Destapando la caja de Pandora académica. Granada: Universidad de Granada.

» O'Reilly, T. 2005. What Is Web 2.0 [O'Reilly]. <http://www.oreilly.com/pub/a/ web2/archive/what-is-web-20.html> [Consulta: 5 diciembre 2016].

"Priem, J. 2010. I like the term \# articlelevelmetrics, but it fails to imply "diversity" of measures. Lately, l'm liking \# altmetrics. [microblog]. <https://twitter.com/jasonpriem/status/25844968813?lang=es > [Consulta: 2 diciembre 2016].

"QS Top Universities. 2015. <http://www.topuniversities.com/universities/universidad-de-la-sabana> [Consulta: 29 julio 2016].

»Redalyc, Grupo de Investigación. 2008. Sistema de información científica Redalyc: la ciencia que no se ve no existe. Toluca: UAEM. <http://ri.uaemex.mx/ handle/20.500.11799/6545> [Consulta: 29 julio 2016].

»Torres-Salinas, D. y Y. Milanés-Guisado. 2014. Presencia en redes sociales y altmétricas de los principales autores de la revista" El Profesional de la Información”. En El Profesional de la Información. Vol. 23, no. 4. <http://digibug.ugr. es/handle/10481/32932> Doi: 10.3145/epi.2014.jul.04 [Consulta: 29 diciembre 2017].

» Turpo, J. E. y G. E. Medina. 2013. Producción intelectual y visibilidad científica. En Apuntes Universitarios. Vol. 2, 9-18. 
